\title{
Noninvasive in vivo Assessment of the Skeletal Muscle and Small Intestine Serous Surface Microcirculation in Rat: Sidestream Dark-Field (SDF) Imaging
}

\author{
Z. TUREK, V. ČERNÝ, R. PǍ̌ÍZKOVÁ \\ Department of Anesthesiology and Intensive Care Medicine, University Hospital Hradec Králové, \\ Hradec Králové, Czech Republic
}

Received November 7, 2006

Accepted February 6, 2007

On-line April 25, 2007

\begin{abstract}
Summary
The pathophysiology of microcirculation is intensively investigated to understand disease development at the microscopic level. Orthogonal polarization spectral (OPS) imaging and its successor sidestream dark-field (SDF) imaging are relatively new noninvasive optical techniques allowing direct visualization of microcirculation in both clinical and experimental studies. The goal of this experimental study was to describe basic microcirculatory parameters of skeletal muscle and ileal serous surface microcirculation in the rat using SDF imaging and to standardize the technical aspects of the protocol. Interindividual variability in functional capillary density (FCD) and small vessels ( $<25 \mu \mathrm{m}$ in diameter) proportion was determined in anesthetized rats on the surface of quadriceps femoris ( $\mathrm{m}$. rectus femoris and $\mathrm{m}$. vastus medialis) and serous surface of ileum. Special custom made flexible arm was used to fix the SDF probe minimizing the pressure movement artifacts. Clear high contrast images were analyzed off-line. The mean FCD obtained from the surface of skeletal muscle and ileal serous surface was 219 (213-225 $\left.\mathrm{cm} / \mathrm{cm}^{2}\right)$ and $290\left(282-298 \mathrm{~cm} / \mathrm{cm}^{2}\right)$ respectively. There was no statistically significant difference between rats in mean values of FCD obtained from the muscle $(P=0.273)$ in contrast to ileal serous surface, where such difference was statistically significant $(P=0.036)$. No statistically significant differences in small vessels percentage was detected on either the muscle surface $(P=0.739)$ or on ileal serous surface $(P=0.659)$. Our study has shown that interindividual variability of basic microcirculatory parameters in rat skeletal muscle and ileum is acceptable when using SDF imaging technique according to a highly standardized protocol and with appropriate fixation device. SDF imaging represents promising technology for experimental and clinical studies.
\end{abstract}

\section{Key words}

SDF imaging • Microcirculation • Functional capillary density • Ileum • Skeletal muscle

\section{Corresponding author}

Zdenek Turek, University Hospital Hradec Králové, Department of Anesthesiology and Intensive Care Medicine, Sokolska 581, 50005 Hradec Králové, Czech Republic. E-mail: turek@fnhk.cz

\section{Introduction}

Microcirculatory level of the cardiovascular system represents a unique environment acting as the site of gas and nutrient exchange between blood and tissues. Analysis of microcirculatory physiology and pathophysiology in various clinical states can provide a feasible opportunity to study disease development. Despite the key role of microcirculation in numerous diseases including sepsis or multiple organ failure (Lehr et al. 2000, Spronk et al. 2004), current available methods allowing objective quantitative assessment of microcirculatory condition applicable to clinical practice are very limited. At present, a great effort has been made to develop and validate new noninvasive methods and technologies for direct assessment of microcirculation.

Techniques for direct visualization and quantitative analysis of microcirculatory flow available at the bedside were reviewed by De Backer and Dubois (2001). Orthogonal polarization spectral (OPS) imaging and its successor sidestream dark-field (SDF) imaging are relatively new, progressively developing noninvasive methods based on very similar principles, which have been described in detail previously (Groner et al. 1999, Schiessler et al. 2002, De Backer 2003, Ince 2005a,b). Briefly, in OPS imaging green $(550 \pm 70 \mathrm{~nm})$ polarized light, guided through a system of lenses, illuminates the 
target tissue to achieve optimal imaging of microcirculation because at this wavelength oxy- and deoxy-hemoglobin absorb the light equally. The light reflected at the surface is eliminated by orthogonal polarizer (analyzer) and does not form an image. The light after undergoing multiple scattering becomes depolarized and passes through the polarizer to create an image of the underlying microcirculation. SDF imaging is based on slightly different principles as compared with OPS technology. Light-emitting diodes (LEDs) arranged in a ring formation at the tip of the light guide emit green light $(540 \pm 50 \mathrm{~nm})$, which directly illuminates the tissue microcirculation. The illuminating light source is optically isolated from the emission light path in the core of the light guide (Fig. 1). Hence, SDF technology provides improved resolution and clarity of the images compared to OPS imaging. Both SDF and OPS technology have been implemented in a hand-held videomicroscope convenient for both experimental and clinical conditions.

Validation studies are essential before employing both optical techniques into clinical practice. Experimental and clinical validation studies have been reviewed recently (Černý et al. 2007). OPS imaging has been validated especially in animal studies by comparison of intravital microscopy (IVM) as a gold standard and OPS techniques. Bland-Altman analysis confirmed good agreement between the two methods applied to both peripheral tissues and solid organs (Langer et al. 2001). Despite further development and improvement of the OPS-SDF imaging methods, several limitations for current clinical practice remain, especially probe pressure movement artifacts and lateral movement of the tissue (Lindert et al. 2002). As mentioned above, SDF imaging is a further enhanced optical method with better clarity, sensitivity and image resolution when compared to OPS imaging (Ince 2005a) and is intended to be used in a wide spectrum of clinical studies (Ince 2005b).

The goal of this experimental study was to describe basic microcirculatory parameters of skeletal muscle and ileal serous surface microcirculation. This is the first study concerning non-invasive microcirculation assessment on the ileal serous surface, which is potentially well accessible during most of intraabdominal surgical procedures. Furthermore, it was designed to elucidate interindividual variability in quantitative assessment of the quadriceps femoris muscle and ileal serous surface microcirculation using SDF imaging in anesthetized spontaneously breathing tracheotomized rats. We hypothesized that interindividual variability of basic microcirculatory parameters such as functional capillary density (FCD) and the proportion of small $(<25 \mu \mathrm{m}$ in diameter $)$ vessels is not statistically significant when using SDF imaging in this experimental study. These data were expected to provide significant information with evident impact on further research based on OPS and SDF imaging technologies both in experimental and clinical studies. This kind of animal study performed by the methodology described below and its relevant findings have not been so far presented in the past.

\section{Methods}

Animals

All experimental procedures were performed after Ethical Board approval in accordance with Czech legislation on the protection of animals. Five male Wistar rats (Bio-Test, Konárovice, Czech Republic), weighing 310-350 g, were included in the study. They were housed in groups of two in a standard cage at $21^{\circ} \mathrm{C}$ in a $12 \mathrm{~h}$ dark/12 h light cycle and were supplied with laboratory chow (Velaz, Prague, Czech Republic) and tap water ad libitum. After one-week acclimatization period, the rats were enrolled into the study.

\section{Anesthesia and surgical preparation}

After overnight fasting with unrestricted access to tap water, the rats were anesthetized with an intraperitoneal induction dose of pentobarbital (Nembutal, Abbott Laboratories, Chicago, IL, USA), $50 \mathrm{mg} / \mathrm{kg}$ of body weight, in case of additional dose $10 \mathrm{mg} / \mathrm{kg}$ of body weight. The animals were placed in a supine position on an operating table; rectal temperature was kept at $36.5-37.5^{\circ} \mathrm{C}$ by use of a heat lamp. The carotid artery and jugular vein were cannulated with polyethylene catheter $24 \mathrm{G}$ for continuous blood pressure monitoring, continuous infusion of normal saline $(20 \mathrm{ml} / \mathrm{kg} / \mathrm{h})$ and to administer additional anesthesia as necessary. Then the animals were tracheotomized (Vasocan 14G, B. Braun Melsungen AG, Germany) in order to maintain patent airway reliably throughout the study.

The abdomen was shaved and opened via a midline laparotomy to exteriorize the ileum. The preparation of the small intestine was performed as described previously (Bohlen and Gore 1976) with slight modifications (Neviere et al. 1999). In brief, the ileocecal 

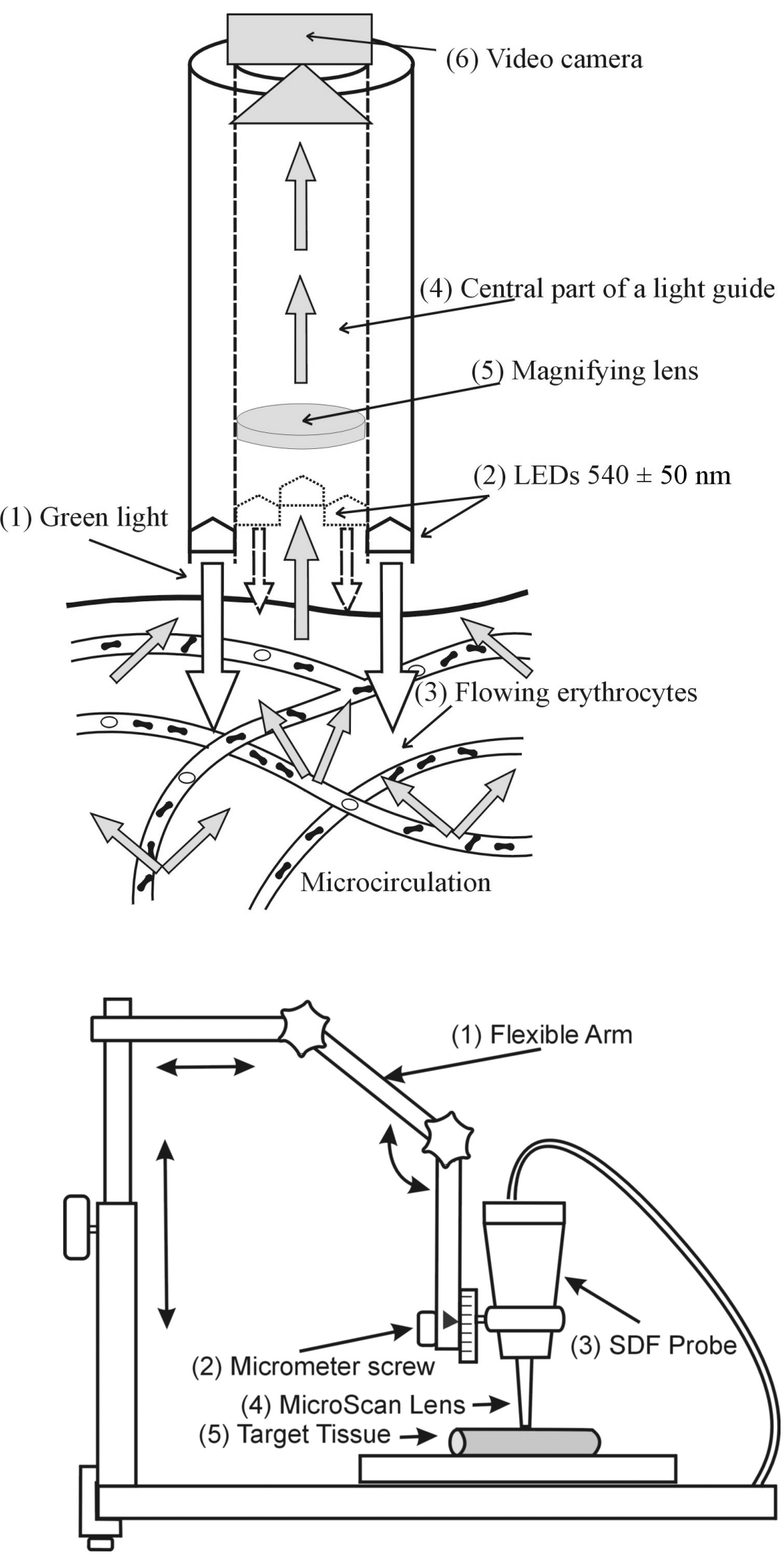

Fig. 1. Sidestream dark-field (SDF) imaging, an optical scheme. (1) Green light is emitted by (2) peripheral $540 \pm$ $50 \mathrm{~nm}$ light-emitting diodes (LEDs) toward tissue arranged in a circle at the end of the light guide. The microcirculation is directly penetrated and illuminated from the side by green light absorbed by hemoglobin of erythrocytes which are observed as (3) dark moving cells. The imaging, central part, of the light guide (4) is optically isolated from the LEDs. A Magnifying lens (5) projects the image onto a camera (6).

Fig. 2. A scheme of the stabilizing and fixation device for SDF imaging probe. (1) Flexible arm allowing horizontal, vertical and rotating movements ends with a special adapter with (2) micrometer screw for movement of the SDF probe (3) with MicroScan lens (4) within the range of $2 \mathrm{~mm}$ towards the target tissue (5). 


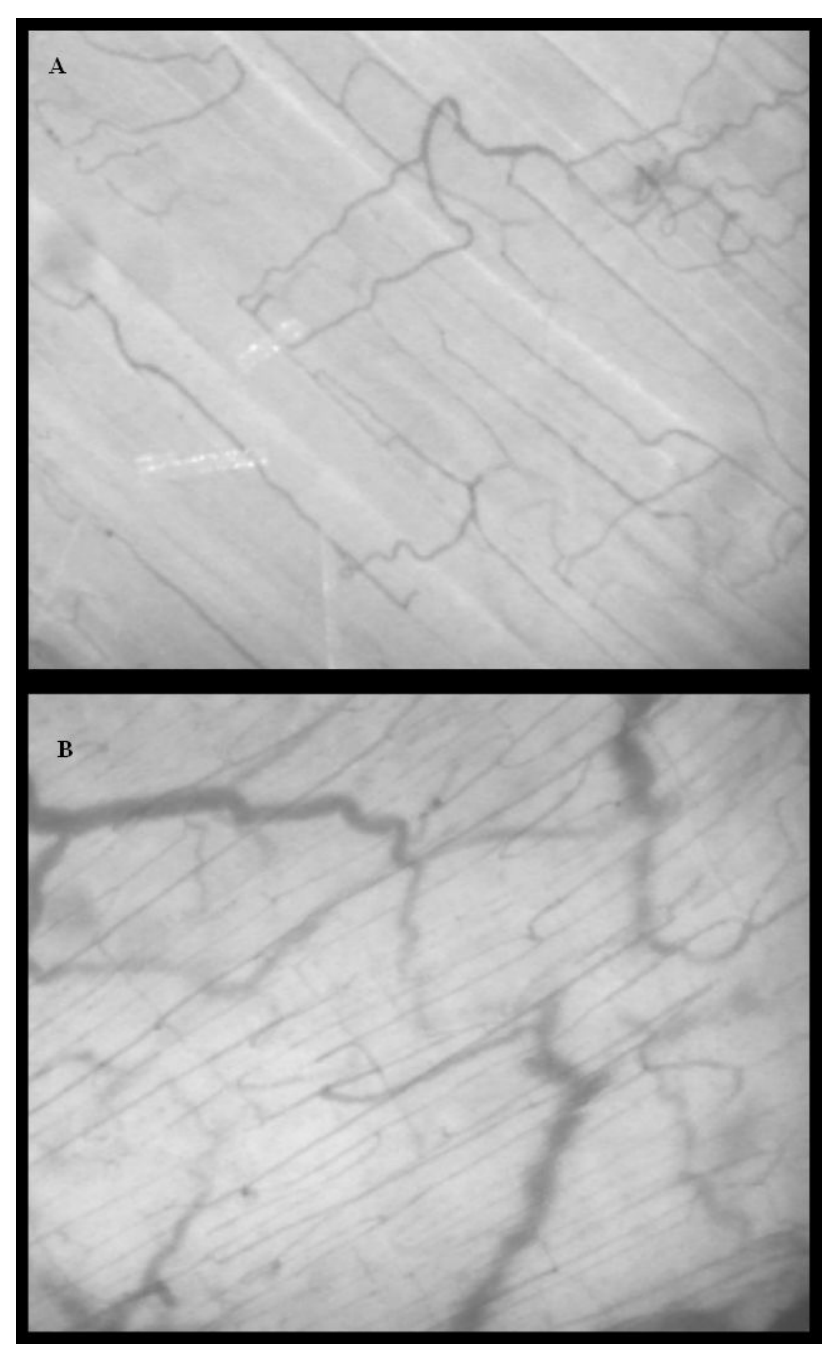

Fig. 3. SDF images of rat quadriceps femoris muscle and ileal serous surface microcirculation. A. Sidestream darkfield (SDF) imaging of the surface of the rat quadriceps femoris muscle. Objective $5 \times$, on screen $325 \times$. B. Sidestream dark-field (SDF) imaging of the ileal serous surface microcirculation. Objective $5 x$, on screen $325 x$.

region was identified and $4 \mathrm{~cm}$ portion of distal ileum along with $5 \mathrm{~cm}$ proximal to ileocecal valve was exposed using warmed normal saline-saturated cotton swabs. The intestinal loop was placed carefully onto a adjustable small plastic plate to avoid breathing movement artifacts and covered with a thin plastic wrap to minimize dehydration (Harris et al. 1998). Any exposed tissues, apart from those covered by SDF imaging probe at the given moment, were intermittently moistened with warmed normal saline at $37{ }^{\circ} \mathrm{C}$. A waiting period of $20 \mathrm{~min}$ followed to confirm the stability of arterial blood pressure and rectal temperature (Langer et al. 2001). Then SDF imaging was performed according to the protocol described below. Once SDF imaging was completed, the ileum was placed back in the abdomen and the skin incision was closed by a suture.

After having shaved the right hindlimb, a craniocaudal skin incision 2-2.5 cm long was performed just in the middle of the medial site of the femur. Then subcutaneous connective tissue was gently prepared, quadriceps femoris muscle $-\mathrm{m}$. rectus femoris and $\mathrm{m}$. vastus medialis were identified and their fascia was carefully separated by blunt dissection from the surface of the muscle in order to minimize tissue injury and bleeding. SDF imaging then resumed according to protocol.

\section{Sidestream dark-field imaging procedure}

To minimize artificial pressure and movement artifacts, the SDF imaging probe (MicroScan Video Microscope, Microvision Medical, Inc., Amsterdam, The Netherlands) was attached to a custom made (Arrow International CR, a.s.) flexible arm with special adapter allowing micromovement of the SDF probe in various axes according to inclination of the flexible arm (Fig. 2). In an effort to objectify the SDF imaging of microcirculation as much as possible, the following methodology was established. Once a sector for imaging was selected, the SDF imaging probe was placed just $1 \mathrm{~mm}$ above the target tissue using a flexible arm. Then the arm was fixed and the probe covered with plastic lens was moved towards tissue by the adapter for micromovement. Immediately after the first contact with the investigated organ, the focus ring of the probe was used to bring the proper layer into focus to create sharp and high contrast image appropriate for later off-line analysis. All SDF imaging data of the microcirculation were digitally recorded. SDF images were obtained from three different areas within the site of interest as recommended recently (Boerma et al.2005). Each area was recorded for a duration of $2 \mathrm{~min}$ and saved. At the end of the experiment the animal was killed by an overdose of pentobarbital.

\section{Off-line analysis}

Off-line selection of the most stable clips with clear images for final analysis was performed. To summarize, a total of nine selected clips (three clips of each area) of 10-15 s were analyzed. The optical magnification was five times, the final on screen magnification of the images obtained with the SDF imaging device was 325 times original and the real size of the field of evaluation was $1280 \mu \mathrm{m}$ x $960 \mu \mathrm{m}$. Basic microcirculatory parameters such as FCD and the 
Tab. 1. Summarized results of basic microcirculatory parameters of each animal

\begin{tabular}{|c|c|c|c|c|c|c|c|c|c|}
\hline & \multicolumn{3}{|c|}{$\operatorname{FCD}\left(\mathbf{c m} / \mathbf{c m}^{2}\right)$} & \multicolumn{3}{|c|}{ Percentage of small vessels (\%) } & \multicolumn{3}{|c|}{ Percentage of medium vessels (\%) } \\
\hline & Mean & $\begin{array}{c}\text { (95 \% C.I. } \\
\text { of mean) }\end{array}$ & SD & Mean & $\begin{array}{c}(95 \% \text { C.I. } \\
\text { of mean) }\end{array}$ & SD & Mean & $\begin{array}{c}(95 \% \text { C.I. } \\
\text { of mean) }\end{array}$ & SD \\
\hline & $\begin{array}{l}\text { Muscle } \\
\text { Ileum }\end{array}$ & $\begin{array}{l}\text { Muscle } \\
\text { Ileum }\end{array}$ & $\begin{array}{l}\text { Muscle } \\
\text { Ileum }\end{array}$ & $\begin{array}{l}\text { Muscle } \\
\text { Ileum }\end{array}$ & $\begin{array}{l}\text { Muscle } \\
\text { Ileum }\end{array}$ & $\begin{array}{l}\text { Muscle } \\
\text { Ileum }\end{array}$ & $\begin{array}{l}\text { Muscle } \\
\text { Ileum }\end{array}$ & $\begin{array}{l}\text { Muscle } \\
\text { Ileum }\end{array}$ & $\begin{array}{l}\text { Muscle } \\
\text { Ileum }\end{array}$ \\
\hline \multirow[t]{2}{*}{ Rat 1} & 196 & $183-205$ & 17.8 & 88.1 & $85.7-90.4$ & 3.1 & 11.8 & $9.4-14.2$ & 3.1 \\
\hline & 256 & $236-286$ & 30.3 & 94.1 & $92.1-96.2$ & 2.7 & 5.6 & $3.5-7.6$ & 2.7 \\
\hline \multirow[t]{2}{*}{ Rat 2} & 201 & $192-210$ & 11.0 & 89.2 & $87.2-91.2$ & 2.6 & 10.2 & $8.1-12.2$ & 2.6 \\
\hline & 253 & $240-265$ & 16.0 & 91.0 & $90.0-92.0$ & 1.3 & 9.8 & $7.8-11.7$ & 2.5 \\
\hline \multirow[t]{2}{*}{ Rat 3} & 204 & $187-215$ & 22.0 & 89.3 & $87.6-91.0$ & 2.2 & 11.7 & $8.6-14.8$ & 4.0 \\
\hline & 264 & $256-272$ & 10.1 & 94.2 & 92.4-95.9 & 2.3 & 6.0 & $4.3-7.8$ & 2.3 \\
\hline \multirow[t]{2}{*}{ Rat 4} & 191 & 184-198 & 9.6 & 88.6 & $87.2-90.0$ & 1.8 & 11.3 & $9.9-12.8$ & 1.8 \\
\hline & 278 & $267-290$ & 20.0 & 91.4 & $89.8-93.0$ & 2.1 & 8.2 & $6.6-9.8$ & 2.1 \\
\hline \multirow[t]{2}{*}{ Rat 5} & 190 & $180-200$ & 12.9 & 88.4 & $86.4-90.6$ & 2.7 & 11.6 & $9.5-13.6$ & 2.7 \\
\hline & 280 & $272-293$ & 16.2 & 93.1 & $91.2-95.1$ & 2.5 & 7.0 & $5.5-8.6$ & 2.0 \\
\hline Summary & 219 & $213-225$ & 16.0 & 88.7 & $88.0-89.5$ & 2.5 & 11.3 & $10.5-12.2$ & 2.9 \\
\hline results & 290 & $282-298$ & 20.5 & 92.8 & $92.0-93.5$ & 2.6 & 7.3 & $6.5-8.2$ & 2.7 \\
\hline
\end{tabular}

FCD (functional capillary density), C.I. (confidence interval), SD (standard deviation)

proportion of small, medium and large vessels were acquired using AVA V1.0 software (AMC, University of Amsterdam, The Netherlands). The following parameters were analyzed off-line:

1) Functional capillary density (FCD) defined as the length of red blood cell-perfused capillaries per observation area and given in $\mathrm{cm} / \mathrm{cm}^{2}$ or $\mu \mathrm{m} / \mu \mathrm{m}^{2}$.

2) Small $(<25 \mu \mathrm{m}$ in diameter) and medium (25$50 \mu \mathrm{m})$ vessels rate $\times 100[\%]$. The diameter in this case is defined semi-quantitatively using presented dimensional constraint. The resulting percentage is ratio [total small vessels length / total length of all analyzed vessels] multiplied by 100 in case of small vessels and [total medium vessels length / total length of all analyzed vessels] multiplied by 100 [\%].

\section{Statistics}

The descriptive data from each animal are presented as mean (95\% confidence interval of mean). One-way ANOVA and Kruskal-Walis one-way ANOVA on ranks were applied to detect interindividual differences in FCD and small vessel percentage within the same organ in various rats - skeletal muscle and ileal serous surface. $P<0.05$ value was considered as statistically significant. All statistical analyses were performed with the use of SIGMASTAT 2.0 (Jandel
Scientific, San Rafael, CA, USA)

\section{Results}

Using special custom-made fixation system for the SDF probe, clear high contrast images were successfully obtained both from the surface of the quadriceps femoris muscle and distal ileum using SDF imaging technology. Typical images of skeletal muscle and ileal serous surface captured using SDF imaging are shown in Figure 3. The mean FCD obtained from the surface of skeletal muscle was $219\left(213-225 \mathrm{~cm} / \mathrm{cm}^{2}\right)$, while the mean FCD on the ileal serous surface was 290 $\left(282-298 \mathrm{~cm} / \mathrm{cm}^{2}\right)$. Calculated small vessel percentage in skeletal muscle and ileal surface was 87.7 (87.0-89.5\%) and 92.8 (91.9-93.5\%) respectively. There was no statistically significant difference between the rats in mean values of FCD obtained from the muscle $(P=0.273)$ in contrast to ileal serous surface where the difference was statistically significant $(P=0.036)$. Interindividual differences in small vessel percentage in muscle and ileal serous surface were also analyzed. Statistically significant differences in small vessel proportion was detected neither on the muscle surface $(P=0.739)$ nor on ileal serous surface $(P=0.659)$. (Table 1). 


\section{Discussion}

SDF imaging allows direct in vivo observation of the rat skeletal muscle and ileal serous surface microcirculation. It was possible to perform basic quantitative measurement of microcirculatory parameters under baseline conditions in rats subjected to a stable general anesthesia. There were two main reasons for the selection of quadriceps muscle and serous surface of the ileum. First, size and accessibility of both organs with minimal tissue injury affecting the microcirculation and second, serous surface of the intestine is very easily accessible during most major abdominal operations. Thus there is a possibility to detect possible splanchnic hypoperfusion in clinical practice using this noninvasive optical technique peroperatively in the future. Furthermore, a significant part of the clinical studies working with OPS and SDF imaging techniques have been performed under general anesthesia during surgical procedures (Mathura et al. 2001, Pennings et al. 2004, van den Oever et al. 2006). The proportion of small vessels was calculated because this parameter can be different in various pathological states characterized by compromised perfusion.

FCD values obtained on the surface of qudriceps muscle are comparable with those obtained from the rat gracilis muscle using IVM previously (Prewitt et al. 1984). The quadriceps muscle has been chosen because of its size in respect of the size of the SDF probe. Similar data from the ileal serous surface have not been published yet. In this study, we have demonstrated that interindividual differences between the rats within the same organ in FCD and small vessels percentage on the quadriceps muscle surface were not statistically significant. Only the differences in mean values of FCD obtained from ileal surface, where the FCD is relatively higher, were statistically significant, but small vessel rate analysis has shown no significant interindividual difference. This fact can be caused by the primary selection of the visualized sector of the ileal surface concerning the presence of large vessels in the microscopic field. Another point is that transverse arterioles $(20-50 \mu \mathrm{m}$ in diameter) were calculated in proportion of medium vessels and terminal arterioles were included in the analysis of capillary beds due to the limitation of software analysis of velocities in arterial part of vessel network. The low number of animals in this experiment can also be a factor affecting the results. Another question remains unanswered, whether the significant interindividual difference in FCD between the rats has any clinical importance because $95 \%$ confidence intervals of mean are relatively narrow. These findings should be taken into account when the changes of microcirculatory parameters under various conditions are interpreted. The evaluation of interindividual differences within the same investigated group should be a standard part of data analysis when using OPS or SDF imaging technology.

Despite progressive development of noninvasive optical methods such as OPS and SDF imaging, several limitations have been clearly identified previously (Christ et al. 2000). It is almost impossible to eliminate the application of pressure with the tip of the MicroScan Lens during examination and lateral movement of tissue precludes continuous monitoring of a selected microvascular region. To minimize these effects and to standardize the examination as much as possible, we have used a custom made flexible fixation device, which can be applied easily both in animal and clinical studies. We consider the use of SDF probe without any fixation device allowing micromovement difficult to interpret objectively. Software analysis is another key point in microcirculatory status assessment. Current available software - the CapImage computer program (Klyscz et al. 1997) and AVA V1.0 software allow accurate off-line evaluation of basic microcirculatory parameters but online analysis remains to be a data-intensive technique unavailable for common experimental and clinical practice.

Thanks to its noninvasiveness, a great effort has been made to introduce OPS imaging followed by SDF imaging into clinical practice by visualization of the sublingual microcirculatory network, especially in critically ill patients with sepsis (de Backer et al. 2002, Sakr et al. 2004). Our study has shown that interindividual variability of basic microcirculatory parameters in rat skeletal muscle and ileum is acceptable when using SDF imaging technique according to a highly standardized protocol and with appropriate fixation device. SDF imaging represents promising technology for experimental and clinical studies.

\section{Conflict of Interest}

There is no conflict of interest.

\section{Acknowledgements}

Research project MZO 00179906. We thank to Mr. Radek Stursa from Arrow International CR, a.s., who invented and made a fixation device for the SDF probe. 


\section{References}

BOERMA EC, MATHURA KR, VAN DER VOORT PH, SPRONK PE, INCE C: Quantifying bedside-derived imaging of microcirculatory abnormalities in septic patients: a prospective validation study. Crit Care 9: R601-R606, 2005.

BOHLEN HG, GORE RW: Preparation of rat intestinal muscle and mucosa for quantitative microcirculatory studies. Microvas Res 11: 103-110, 1976.

ČERNÝ V, TUREK Z, PAŘÍZKOVÁ R: Orthogonal polarization spectral imaging. Physiol Res 56: 141-147, 2007.

CHRIST F, GENZEL-BOROVICZENY O, SCHAUDIG S, NIKLAS M, SCHIESSLER C, STROTGEN J, EIFERT S, REICHENSPURNER H, HARRIS AG, MESSMER K: Monitoring of the microcirculation in cardiac surgery and neonates using orthogonal polarization spectral imaging. In: Orthogonal Polarization Spectral Imaging. MESSMER K (ed), Karger, Basel, 24: 82-93, 2000.

DE BACKER D: OPS techniques. Minerva Anesthesiol 69: 388-391, 2003.

DE BACKER D, DUBOIS MJ: Assessment of the microcirculatory flow in patients in the intensive care unit. Curr Opin Crit Care 7: 200-203, 2001.

DE BACKER D, CRETEUR J, PREISER JC, DUBOIS MJ, VINCENT JL: Microvascular blood flow is altered in patients with sepsis. Am J Respir Crit Care Med 166: 98-104, 2002.

GRONER W, WINKELMAN JW, HARRIS AG, INCE C, BOUMA GJ, MESSMER K, NADEAU RG: Orthogonal polarization spectral imaging: a new method for study of the microcirculation. Nat Med 5: 1209-1212, 1999.

HARRIS AG, COSTA JJ, DELANO FA, ZWEIFACH BW, SCHMID-SCHONBEIN GW: Mechanisms of cell injury in rat mesentery and cremaster muscle. Am J Physiol 274: H1009-H1015, 1998.

INCE C: Sidestream dark-field (SDF) imaging: an improved technique to observe sublingual microcirculation. Crit Care 8 (Suppl): P72, 2005a.

INCE C: The microcirculation is the motor of sepsis. Crit Care 9 (Suppl 4): S13-S19, 2005 b.

KLYSCZ T, JUNGER M, JUNG F, ZEINTL H: Cap image - a new kind of computer-assisted video image analysis system for dynamic capillary microscopy. Biomed Tech (Berl) 42: 168, 1997.

LANGER S, HARRIS AG, BIBERTHALER P, VON DOBSCHUETZ E, MESSMER K: Orthogonal polarization spectral imaging as a tool for the assessment of hepatic microcirculation. Transplantation 71: 1249-1256, 2001.

LEHR HA, BITTINGER F, KIRKPATRICK CJ: Microcirculatory dysfunction in sepsis: a pathogenetic basis for therapy? J Pathol 190: 373-386, 2000.

LINDERT J, WERNER J, REDLIN M, KUPPE H, HABAZETTL H, PRIES AR: OPS imaging of human circulation: a short technical report. J Vasc Res 39: 368-372, 2002.

MATHURA KR, BOUMA GJ, INCE C: Abnormal microcirculation in brain tumors during surgery. Lancet 358: 16981699, 2001.

NEVIERE RR, PITT-HYDE ML, PIPER RD, SIBBALD WJ, POTTER RF: Microvascular perfusion deficits are not a prerequisite for mucosal injury in septic rats. Am J Physiol 276: G933-G940, 1999.

VAN DEN OEVER HL, DZOLJIC M, INCE C, HOLLMANN MW, MOKKEN FC: Orthogonal polarization spectral imaging of the microcirculation during acute hypervolemic hemodilution and epidural lidocaine injection. Anesth Analg 103: 484-487, 2006.

PENNINGS FA, BOUMA GJ, INCE C: Direct observation of the human cerebral microcirculation during aneurysm surgery reveals increased arteriolar contractility. Stroke 35: 1284-1288, 2004.

PREWITT RL, CHEN II, DOWELL RF: Microvascular alterations in the one-kidney, one-clip renal hypertensive rat. Am J Physiol 246: H728-H732, 1984.

SAKR Y, DUBOIS MJ, DE BACKER D, CRETEUR J, VINCENT JL: Persistent microvasculatory alterations are associated with organ failure and death in patients with septic shock. Crit Care Med 32: 1825-1831, 2004.

SCHIESSLER C, SCHAUDIG S, HARRIS AG, CHRIST F: Orthogonal polarization spectral imaging - a new clinical method for monitoring of microcirculation. Anaesthesist 51: 576-579, 2002.

SPRONK PE, ZANDSTRA DF, INCE C: Bench-to-bedside review: sepsis is a disease of microcirculation. Crit Care 8: 462-468, 2004. 